\title{
DIAGNOSTIC YIELD OF DYNAMIC CONTRAST ENHANCED MR IMAGING IN BREAST LESIONS, WHICH ARE INDETERMINATE ON CONVENTIONAL AND ULTRASOUND MAMMOGRAPHY
}

\author{
Beenamol S1, John N. J2 Saanida M. P3, Ramachandran $K^{4}$ \\ ${ }^{1}$ Associate Professor, Department of Radiodiagnosis, Government Medical College, Trivandrum. \\ ${ }^{2}$ Associate Professor, Department of Radiodiagnosis, Government Medical College, Trivandrum. \\ ${ }^{3}$ Assistant Professor, Department of Radiodiagnosis, Government Medical College, Calicut. \\ 4 Professor and HOD, Imageology Division, Regional Cancer Centre, Trivandrum.
}

\section{ABSTRACT}

\section{BACKGROUND}

Though mammography remains the imaging of choice in evaluating breast lesions, it has a relatively high false positivity and false negativity. It is relevant to find a superior alternative in case of indeterminate mammographic results.

The objective of the study is to compare the diagnostic yield of Magnetic Resonance Mammogram with that of a combination of $\mathrm{x}$-ray mammogram and sonomammogram in differentiating benign and malignant breast lesions.

\section{MATERIALS AND METHODS}

This study was done at the Regional Cancer Centre, Trivandrum and Government Medical College, Trivandrum over a 1-year period. Study population included the patients with breast lumps referred for Magnetic Resonance Imaging, who were investigated by mammography and sonography and diagnosed to have indeterminate imaging findings. Sample size was 50 . Study design was observation with diagnostic test evaluation. The final pathological diagnosis in all patients was confirmed by biopsy and histopathological examination.

\section{RESULTS}

Of the 50 cases studied 28 were found to be malignant, 19 were found to be benign; 10 cases showed no mass on precontrast imaging; 7 of them showed non-mass like enhancement and there were no mass on post-contrast imaging in 3 cases. The overall sensitivity was $78 \%$ and specificity was $63 \%$ in detecting malignancy.

\section{CONCLUSION}

The diagnostic yield of MR imaging in breast lesions is superior to that of a combination of mammography and sonography. MR imaging is more sensitive to malignancy than any imaging modality alone and can be used as a problem solving study when there is diagnostic dilemma with routine mammography and sonomammography.

\section{KEYWORDS}

Breast Cancer, Breast MRI, Mammography, MR Mammography, Sonomammography.

HOW TO CITE THIS ARTICLE: Beenamol S, John NJ, Saanida MP, et al. Diagnostic yield of dynamic contrast enhanced MR imaging in breast lesions, which are indeterminate on conventional and ultrasound mammography. J. Evolution Med. Dent. Sci. 2017;6(5): 375-381, DOI: $10.14260 /$ Jemds/2017/85

\section{BACKGROUND}

X-ray mammography has been in clinical use for the last few decades and is an established tool in the diagnosis of malignant breast lesions. However, x-ray mammography has well recognised limitations. It has a false negative rate between $4-34 \%$. It uses ionising radiation and there are some reports that routine $\mathrm{x}$-ray mammogram itself increases the incidence of malignancy due to the radiation involved. Addition of sonomammography has improved the pick-up rate of malignant lesions.

MRI (Magnetic Resonance Imaging) mammography is not yet widely accepted as a routine test in the diagnostic investigation of breast lesions. The absence of ionising radiation in the MR study is an advantage. The high cost of MR mammography and accessibility are known

Financial or Other, Competing Interest: None.

Submission 20-12-2016, Peer Review 03-01-2017,

Acceptance 07-01-2017, Published 16-01-2017.

Corresponding Author:

Dr. Beenamol S.,

V. P. House, Neerkunnam East,

Vandanam P O, Alleppey-5, Kerala.

E-mail: beenamols@gmail.com

DOI: $10.14260 /$ jemds $/ 2017 / 85$ disadvantages. But demonstration of a definite advantage of MR over combination of x-ray mammography and sonomammography may point to the inclusion of MR mammography in the group of investigations for indeterminate breast lesions.

\section{MATERIALS AND METHODS}

This was a prospective study with diagnostic test evaluation design. The study population included a total of 50 patients in all age groups referred for MRI to the Department of Imageology, Regional Cancer Centre, Trivandrum and Government Medical College, Trivandrum, for a period of twelve months. Patients with inconclusive study by combined mammography and ultrasound and with palpable breast lumps were included in the study. Patients under hormone therapy, patients in luteal phase of menstrual cycle, patients who are unable to lie prone to be positioned within the breast coil, patients whose breasts cannot be accommodated within the breast coil, patients with usual contraindications to MRI like cardiac pacemaker, cochlear implants and other metallic objects within the body, patients not willing for FNAC and patients not giving consent to participate in the study were excluded from the study. 


\section{Imaging Technique}

Using 1.5 Tesla (Signa HDXt) MRI machine manufactured by GE systems using a dedicated bilateral phased array breast coil, MR imaging was performed with dedicated breast surface coils. Most of the breast coils are designed such that patients lie prone with the breasts dependent within the coil. Some coils are equipped with the capability of applying compression to the breast. The advantages of compression include minimization of patient motion and decrease in thickness of the breast with a subsequent decrease in image acquisition time.

\section{Imaging Protocol}

A comprehensive dynamic protocol instead of fast dynamic imaging in the first 4 seconds followed by slow dynamic imaging in 5 consecutive series at 78 second intervals. The protocol started with an unenhanced non-fat suppressed T2W axial Fast Spin-Echo images and a full $512 \times 512$ imaging matrix (TR/TE 7000/60. A TIW 3D fat suppressed fast spoiled Gradient Echo (6.2/3) flip angle 10, band width 31.25 $\mathrm{MHz}$ sequence was then performed before and 6 times after a rapid bolus injection of $1 \mathrm{mmol} / \mathrm{L}$ of Gadopentetate Dimeglumine per kilogram of body delivered through an indwelling IV catheter. Image acquisition started after contrast material injection and saline bolus. Images were obtained sagittally for an acquisition time per volumetric acquisition of less than 2 mins. (1.18 sec) each.

Section thickness was $2.6 \mathrm{~mm}$ without a gap, using a matrix of $256 \times 160$ and a field of view of $20-23 \mathrm{cms}$. Frequency was in the antero-posterior direction. After the examination, the unenhanced images were subtracted from the first enhanced images on a pixel by pixel basis. It is calculated using the formula,

Initial signal increase (\%)

\begin{tabular}{|l|}
\hline Signal post $\mathrm{cm}-$ Signal pre $\mathrm{cm}$ \\
\hline Signal pre $\mathrm{cm}$
\end{tabular}

\section{Initial Signal Increase}

None to Slight - Less than $50 \%$ increase in signal intensity compared to pre-contrast.

Moderate - Between $50 \%$ and $100 \%$ increase in signal intensity compared to pre-contrast.

Strong - Over $100 \%$ increase in signal intensity compared to pre-contrast.

Post initial signal behaviour.

This describes the course of signal curve between $2-8$ minutes after contrast administration.

\section{Post initial signal behaviour $=$}

\begin{tabular}{|l|}
\hline Signal $8 \mathrm{mts}-$ Signal (max $1-2 \mathrm{mts})$ \\
$\frac{\text { Signal }(\max 1-2 \mathrm{mts})}{\mathrm{x}} 100 \%$ \\
\hline
\end{tabular}

Three Types of Time Signal Intensity Curves have been Described

Continuous - Signal increase over $10 \%$.

Plateau - Constant signal intensity (+/- 10\%).

Washout - Signal decrease over 10\%.

The Following Variables are Assessed for A Lesion
A. Shape, B. Margin, C. T2 Characteristics, D. Perilesional Oedema, E. Septa, D. Enhancement, E. Dynamic study

A. Initial Peak.

C1 $-<50 \%$.

C2 - $50-100 \%$.

C3 - > 100\%.

B. SI 3 - 8 minutes post-contrast.

Continued - Type I curve.

Plateau - Type II curve.

Washout - Type III curve.

F. Lymph node size, $1 .<1 \mathrm{~cm}, 2 .>1 \mathrm{~cm}$.

Comparison between pre-contrast and post-contrast sequences, subtraction and maximal intensity projection images are all valuable tools in interpretation. Enhancing lesions of the breast on MRI can be evaluated by morphology, enhancement kinetics or both.

Sequences like diffusion weighted imaging and spectroscopy are not included in the present study.

\section{Morphologic Criteria}

Morphologic criteria to be studied for MR characterisation include distribution, shape, margins and internal enhancement characteristics. Morphologic features associated with malignancy on MR imaging examination are irregular shape and irregular or spiculated margins [Figure 1]. In addition to rim enhancement, heterogeneous, internal enhancement and ductal distribution are features associated with a relatively high positive predictive value. Smooth margins and homogeneous internal enhancement are often associated with benign lesions [Figure 2].

\section{Enhancement Criteria}

There are basically three types of enhancement curves associated with breast lesions seen on MR imaging. These curves access the initial slope of enhancement occurring generally within the first 2 mins. after contrast administration and later enhancement behaviour. In the Type 1 or progressive curve enhancement continues to increase with each post-contrast sequence [Figure 4]. In Type 2 or plateau curve, enhancement levels off after the first postcontrast scan [Figure 5]. In the Type 3 or wash out curve enhancement decreases after the initial rise [Figure 6]. Type 1 curve is usually associated with benign entities and Type 3 with malignancy.

The report was written according to the set pattern and were entered in the proforma for further analysis. The data are analysed using SPSS and various indices like sensitivity, specificity, positive predictive value, negative predictive value, likelihood ratio+ and likelihood ratio- are calculated. The final pathological diagnosis in all patients were confirmed by biopsy or FNAC.

\section{RESULTS}

A total number of 50 cases were studied. All these patients had initial clinical examination and combined mammography and ultrasound scanning as well as pre- and post-contrast MR imaging. All these patients had histological examination. Histopathological report was considered as the gold standard. Of these 33 cases were of inconclusive diagnosis by combined mammography and sonography. 
11 cases were for preoperative assessment and 6 cases were suspected benign but inconclusive by mammogram; 10 cases showed no mass on pre-contrast imaging. These cases were excluded from the statistical calculations. Of the 10 cases, there were no mass on post-contrast imaging in 3 cases; 7 cases showed non-mass like enhancement.

16 patients were of the age group $30-39$ yrs. and 24 were between 40 - 49 yrs. Indications for MR imaging were inconclusive study by combined mammography and sonography, pre-operative assessment prior to breast conservation surgery to detect recurrence/residual lesion in post-surgery and post-chemotherapy cases, large lesions identified as benign in dense breasts and high risk cases (Patients with history of unilateral mastectomy for malignancy). In calculations, post-chemotherapy postsurgery cases were included among the inconclusive lesions. Hence, inconclusive cases were 33 [66\%], preoperative cases were 11 [22\%] and suspected benign but inconclusive by mammography 6 [12\%]. Of the total 28 cases [56\%] were malignant, 19 [38\%] were benign and 3 [6\%] were negative for disease. Of the 33 cases inconclusive by mammography, 19 cases were positive for malignancy and 12 were benign and 2 were negative. Of the total cases, 3 were negative for mass and 47 showed mass; 27 cases were IDC with DCIS and 1 was DCIS. Of the 19 benign cases, 5 were fibroadenomas, 4 were benign intraductal hyperplasia, 7 papillomas, 2 sclerosing adenosis and 1 inflammatory mass.

\begin{tabular}{|c|c|c|c|c|c|c|c|c|c|}
\hline Index & OS & RS & LS & SM & T2 Hy & HE & IP & T 1C & OS+SM+T 1C \\
\hline Sensitivity & 27.78 & 22.22 & 33.33 & 77.77 & 77.77 & 61.11 & 55.5 & 72 & 27.7 \\
\hline Specificity & 95.45 & 95.4545 & 90.9090 & 100 & 77.27 & 95.45 & 100 & 100 & 100 \\
\hline Positive Predictive Value & 83.33 & 80 & 75 & 100 & 73 & 91.66 & 100 & 100 & 100 \\
\hline Negative Predictive Value & 61.76 & 60 & 62.5 & 84.615 & 80 & 75 & 73.3 & 81 & 62.8 \\
\hline Positive Likelihood Ratio & 0.29 & .21 & 0.37 & .78 & 1.01 & 0.645 & 0.56 & .72 & .28 \\
\hline Negative Likelihood Ratio & 0. & .2 & .035 & .76 & 0.98 & 0.62 & 0.54 & .71 & .26 \\
\hline
\end{tabular}

OS - Oval Shape.

RS - Round Shape.

LS - Lobulated Shape.

SM - Smooth Margins.

T2 Hy - T2 Hyperintensity.

HE - T2 Homogeneous enhancement.

IP - T2 Initial Peak $<50$.

T 1C - Type 1 curve.

OS+SM+T 1C - Oval Shape + Smooth Margin + Type 1 Curve.

\begin{tabular}{|c|c|c|c|c|c|c|c|}
\hline Index & SM & T2 Hy & HE & CE & IP & T 3C & IS+SM+T 3C \\
\hline Sensitivity & 86.36 & 68.18 & 50 & 22.72 & 100 & 95.45 & 59.09 \\
\hline Specificity & 100 & 72.22 & 78.33 & 100 & 50 & 94.44 & 100 \\
\hline $\begin{array}{c}\text { Positive } \\
\text { Predictive Value }\end{array}$ & 100 & 75 & 78.57 & 100 & 70.96 & 95.45 & 100 \\
\hline $\begin{array}{c}\text { Negative } \\
\text { Predictive Value }\end{array}$ & 85.71 & 65 & 57.69 & 22.8 & 100 & 94.44 & 66.66 \\
\hline $\begin{array}{c}\text { Positive } \\
\text { Likelihood Ratio }\end{array}$ & 0.87 & 0.93 & 0.6 & 0.23 & 2 & 1.02 & 0.59 \\
\hline $\begin{array}{c}\text { Negative } \\
\text { Likelihood Ratio }\end{array}$ & 0.85 & 0.95 & 0.58 & 0.22 & 1.98 & 1 & 0.58 \\
\hline
\end{tabular}

SM - Spiculated Margins.

T2 Hy - T2 Hypointensity.

HE - T2 Heterogeneous enhancement.

CE - Clumped Enhancement.

IP - T2 Initial Peak > 100.

T 3C - Type 3 Curve.

IS+SM+T 3C - Irregular Shape + Spiculated Margin + Type 3 Curve

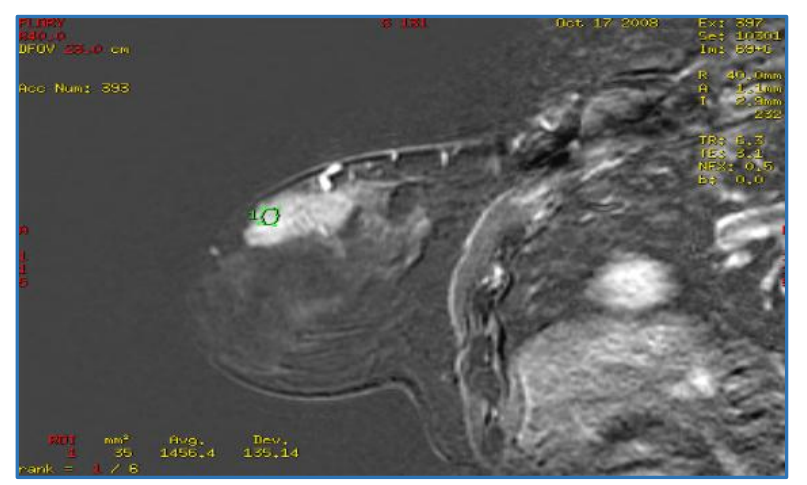

Figure 1. MRI Image - Irregular Shape of Infiltrating Ductal Carcinoma 


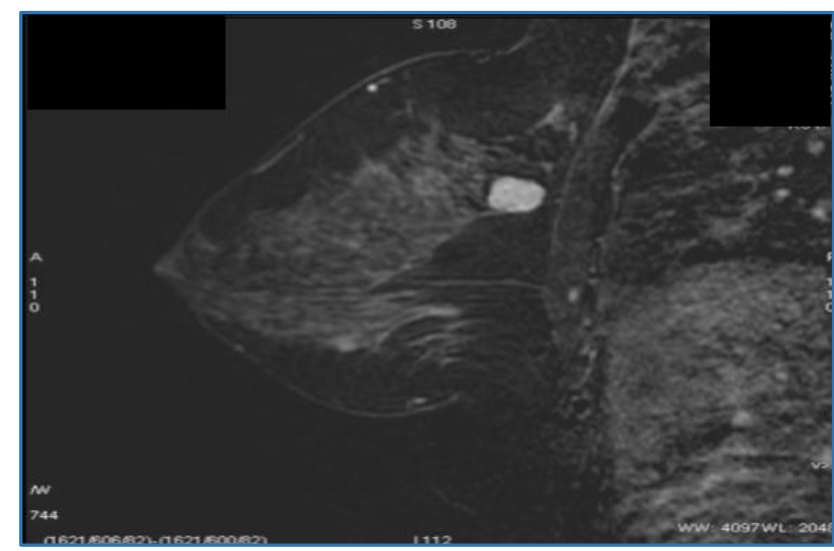

Figure 2. MRI Image - Smooth Margins and T2 Hyperintensity of Fibroadenoma

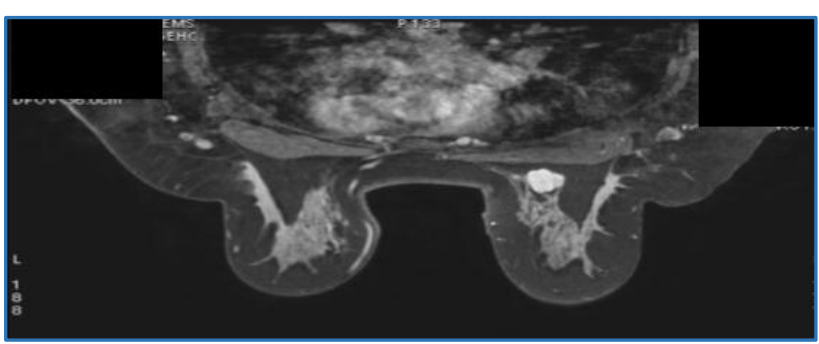

Figure 3. MRI Image - Spiculated Margins of Infiltrating Ductal Carcinoma

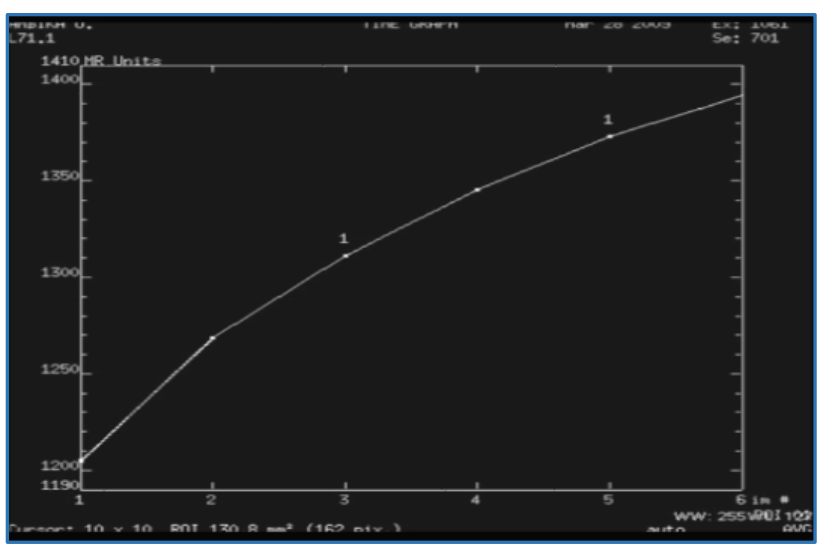

Figure 4. Continuous Curve - Fibroadenoma

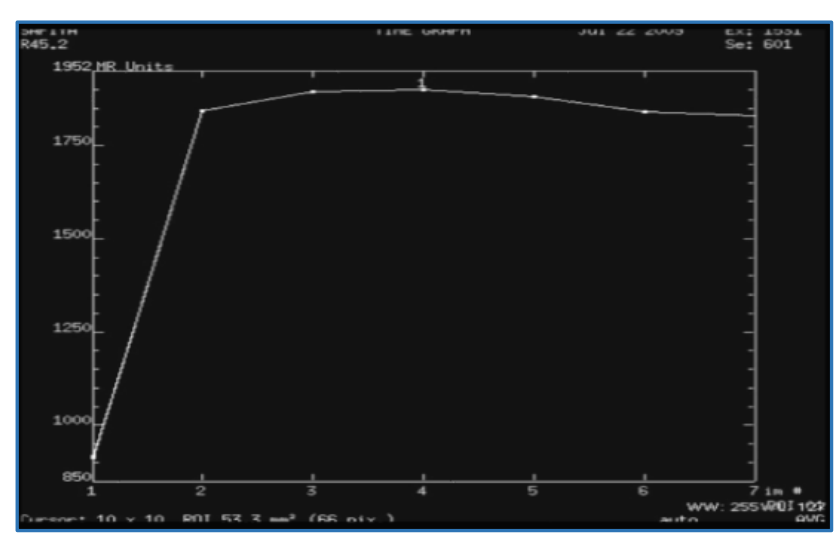

Figure 5. Plateau Curve - Papilloma

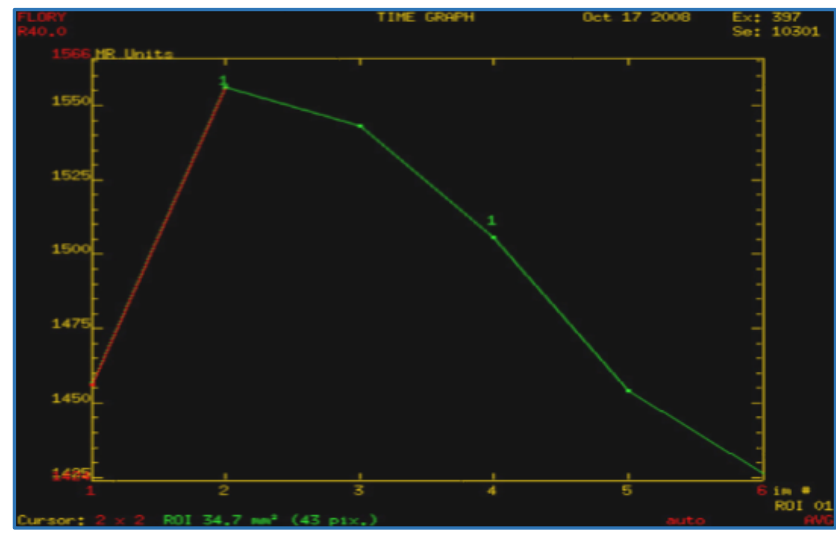

Figure 6. Washout Curve - Infiltrating Ductal Carcinoma

\section{DISCUSSION}

Detection of breast cancer is the primary goal of breast imaging. Mammography has proved effective as a screening test for the early detection of breast cancer. In dense and heterogeneously dense breasts, mammographic sensitivity is decreased 1,2 with as few as $30 \%-48 \%$ of cancers depicted in extremely dense breasts. Indeed in this study, where $50 \%$ of cancers were palpable, mammographic sensitivity was poor in extremely dense breasts and in heterogeneously dense breasts and was lower for IDC across all grades of breast density. In this study, cancer was nearly twice as prevalent in dense or heterogeneously dense breasts than in non-dense breasts grades.

The combination of decreased mammographic sensitivity and increased prevalence of cancer in denser breasts ${ }^{3}$ has prompted interest in the investigation of supplemental screening with US or even MR imaging. Stomper et al $^{4}$ reported that the frequency of dense breasts decreases with increasing age with $62 \%$ of women in their 30 s having more than $50 \%$ breast density compared with $27 \%$ of women in their 60s. Several studies have shown decreased mammographic sensitivity in younger women even after correcting for breast density. Mammographically, occult tumour $^{5}$ may help explain the observations that women younger than 40 years with positive margins are at increased risk of having residual tumour in their breasts compared with older women and that women younger than 40 years' experience a much higher rate of local recurrence after radiation therapy than do older women, even with a boost to the lumpectomy site.

As summarised in the study of Lieberman et al $^{6}$ across multiple series totalling 1280 breasts with detailed after a preoperative diagnosis of unifocal cancer, 619 (48\%) were found to have additional tumour foci unsuspected on mammography. In this study, 2 cases in which conservation or no surgery was anticipated after mammography and clinical examination, additional lesions were detected which were mammographically occult. Preoperative imaging was to assess location, extent, multi-focality and multi-centricity 72 cases showed disease in the opposite breast.

In the studied sample number, size and extent correlated well with operative findings. Supplemental MR imaging depicted additional tumour that necessitated wider excision in 1 case for which conservation was anticipated. Liberman et al ${ }^{6}$ reported additional tumour depiction with MR imaging in $27 \%$ of ipsilateral breasts. Orel et al ${ }^{8}$ reported additional tumour depiction with MR imaging in $34 \%$ of patients, the 
vast majority of additional tumour foci are found in the same quadrant as the index lesion. Thus, the vast majority of additional tumour foci are in the same quadrant and within 4 $\mathrm{cm}$ of the index cancer. At a minimum, when performing USS in a patient known or suspected to have cancer, a thorough evaluation of at least that quadrant of the breast is recommended. Evaluation of the contralateral breast deserves discussion. 9,10

On the basis of clinical or mammographic evaluation or both, $2 \%-3 \%$ of patients are found to have synchronous bilateral cancer. Invasive lobular histologic findings and age younger than 55 years increase the risk of contralateral breast cancer. In the present study $2 \%$ of patients had synchronous bilateral cancer with $3 \%$ of patients identified as having unsuspected contralateral cancer at US and MR imaging that was occult at mammography and clinical examination. Similar rates of $4 \%-6 \%$ of unsuspected contralateral breast cancer have been seen in other series on the basis of supplemental imaging beyond mammography. A benefit of MR imaging over and above combined mammography, clinical examination and US in 2 out of 11 (2\%) breasts for which conservation (or no surgery) had been planned, similar to the 7\% (seven of 104) additional benefit of MR imaging in the series of Hlawatsch et al. ${ }^{11}$

In five cases of IDC with an additional MR imaging benefit, MR imaging depicted an extensive intraductal component that was otherwise occult; presumably, these patients would have had positive margins at lumpectomy. Ideally, patients with EIC could be selected to undergo preoperative MR imaging. Mai et $\mathrm{al}^{12}$ found that EIC could be predicted on the basis of core biopsy findings. If at least three cores had lowgrade DCIS or at least two cores had high-grade DCIS, 20 of $23(87 \%)$ patients with IDC had EIC at excision. Importantly, 9 of $15(60 \%)$ lumpectomies in the group with core biopsy finding suggestive of EIC (According to the criteria of Mai et $\mathrm{al}^{12}$ ) had positive margins or margins less than $1 \mathrm{~mm}$. Further validation of this approach is needed.

In the patient with newly diagnosed ILC, MR imaging was particularly helpful in the assessment of disease extent. Rodenko et al ${ }^{13}$ showed correlation with pathologic extent of disease in 17 of 20 (85\%) MR evaluations compared with only $32 \%$ correlation with mammographic studies. In the series of Weinstein et al,14 MR imaging changed clinical management in $50 \%$ of patients with ILC. In this study, no case of ILC was detected. Liberman et $\mathrm{al}^{6}$ found that additional sites of cancer were more common with lobular than ductal histologic findings and in women with a family history of breast cancer was not significant. Contralateral breast cancer is more common with lobular histologic findings than IDC.

The $25 \%$ - 36\% rate of local recurrence in the absence of radiation or chemotherapy (parallels the frequency of multifocal and multicentric tumour ${ }^{9}$ found only at US and/or MR imaging evaluation. This suggests the potential for these additional tumour foci to be clinically relevant and may also suggest that complete excision directed by multimodality pre-treatment imaging may decrease the need for radiation therapy, though such studies have not yet been performed. Much of the additional tumour that is occult at mammography would be identified at surgery.

Tumour size can be underestimated, but this is often evident at time of surgery or would certainly yield positive margins or mastectomy would be achieved. IDC represents $85 \%-90 \%$ of all invasive carcinoma and ILC represents $10 \%$ - $15 \%$ of invasive breast cancer. From this, we would expect residual tumour in approximately $19 \%$ of patients with invasive carcinoma if management proceeded only on the basis of mammography and clinical examination with standard surgical management, i.e. repeat excision or mastectomy if margins are positive. It is imperative that histopathologic proof of disease extent be obtained preoperatively whenever possible to assist in proper surgical planning.

Non-Mass like Enhancement [NMLE] were noted in 7 cases; 4 cases were malignant and 3 were benign; 3 showed no mass on post-contrast study also. Among the NMLE cases with malignant histology one was Ductal Carcinoma In Situ [DCIS] and 3 were Invasive Ductal Carcinoma [IDC] with DCIS. Invasive ductal carcinoma presents particular dilemmas in the detection and evaluation of disease extent. Of the 3 benign cases, 2 were papillomas and 1 was focal intraductal hyperplasia. Brown et al reported that $29 \%$ of MR imaging studies showed incidental enhancing lesions. Teifke et al ${ }^{15}$ found incidental enhancing lesions in $16 \%$ of patients. The criteria for classification of enhancing lesions found at MR imaging as benign or probably benign are not well established. When contrast material continues to accumulate within the lesion over time, (i.e. progressive or persistent kinetics), this tends to correlate with benignancy. Kuhl et $\mathrm{al}^{16,17}$ found that 9 of $146(6 \%)$ lesions with progressive or initially progressive then delayed plateau enhancement were malignant, and this represented $9 \%$ of malignant lesions in their series.

Detailed morphologic analysis is not readily performed for very small lesions (Smaller than $5 \mathrm{~mm}$ ) when bilateral breast MR imaging is performed because of the requirement for imaging both breasts within the first $1-2$ minutes after contrast material injection and resulting in limited resolution. Experimental contrast agents with prolonged intravascular phase may facilitate detailed morphologic analysis and biopsy of lesions seen only at MR imaging. Liberman et al ${ }^{18}$ found that 9 of $89(10 \%)$ women with lesions considered probably benign at MR imaging proved to have malignancy at a median followup of 11 months, though 3 of 9 (33\%) of the malignancies were at other sites. Prognosis was not likely adversely affected by short interval follow-up in that series, as 5 of $9(56 \%)$ cancers were DCIS, and the median size of the IDCs was $6 \mathrm{~mm}$.

Full assessment of the local extent of breast cancer also includes the axilla. The enhancement of lymph nodes ${ }^{19}$ in the axilla and even (rarely) internal mammary chain was seen at MR imaging. We have found that when a node is identified at MR imaging that enhances more intensely or is visually strikingly larger than adjacent nodes or has lost its fatty hilum that subsequent identification and US-guided fineneedle aspiration biopsy can facilitate surgical planning. Lymph node size of $>1 \mathrm{~cm}$ we noted in 14 cases and all were positive for malignancy. If a nodal metastasis is confirmed, the patient will typically proceed straight to full axillary dissection rather than initial sentinel node biopsy and second axillary surgery if the sentinel node is positive. Refinement of criteria and further validation of this approach are warranted. 
Morphological assessment of the lesions was done on post-contrast imaging. A mass is a 3D lesion that occupies a space within the breast. Masses are described in terms of shape, margin and internal enhancement characteristics. Predictability of benign and malignant lesions was studied with single variable and multiple variables. Shape - A mass can be round, oval, lobulated or irregular. Lobulated masses have an undulating contour. There were 6 cases with oval shape and 8 cases with lobulated shape. The specificity of oval shape for a benign lesion was $95 \%$ and positive predictive value of $83 \%$. Lobulated shape has a specificity of $90 \%$ in diagnosing a benign lesion. Masses with irregular shape had a sensitivity of $81 \%$, specificity of $83 \%$ and positive predictive value of $85 \%$.

Margins can be described as smooth, irregular or spiculated. Spiculated margins frequently are a feature of malignant breast lesions and radial scars. Smooth margin had a sensitivity of $78 \%$, specificity of $100 \%$, PPV of $100 \%$ and NPV of $84.6 \%$ in diagnosing benign lesions. Spiculated margin is essentially a feature of malignancy and it has a sensitivity of $86 \%$, specificity of $100 \%$ and PPV of $100 \%$ and NPV of $84 \%$. Signal characteristics in T2 imaging - T2 hyperintensity has a sensitivity of $77 \%$, specificity of $77 \%$, PPV of $73 \%$ and NPV of $80 \%$ in categorising benign lesions. T2 hypointensity has sensitivity of $68 \%$, specificity of $75 \%$, PPV of $62 \%$ and NPV of $75 \%$ in diagnosing malignant lesions.

Homogeneous enhancement has a sensitivity of $57 \%$, specificity of $100 \%$, PPV of $100 \%$ and an NPV of $74 \%$ in concluding a lesion as benign. ${ }^{20}$ Clumped and heterogeneous enhancement characterise malignant lesions. Clumped enhancement pattern has a specificity of $94 \%$ and PPV of $89 \%$, heterogeneous enhancement pattern has a sensitivity of $38 \%$, specificity of $80 \%$ and PPV of $71 \%$. Stippled enhancement has a specificity and PPV of $100 \%$. Dark internal septations not enhanced within an enhanced lesion are typical of fibroadenomas when the lesion has smooth or lobulated borders. Enhanced internal septations are usually a feature of malignant lesions.

The most suspicious curve pattern derived from the fastest enhancing part of a lesion is chosen to describe the enhancement curve. According to Basak Erguvan-Dogan et al, the initial enhancement phase-enhancement the first 2 minutes after contrast injection or until the curve starts to change is described as slow [<50] medium [50 - 100 or rapid] [ $>100]$. Enhancement $<50$ was favouring malignancy and a sensitivity of $55.5 \%$, specificity of $100 \%$ and PPV of $100 \%$ and NPV of $73.3 \%$ was obtained. In this study, enhancement of 50 - 100 has equal specificity [84\%] for both benign and malignant lesions. Enhancement of $>100$ has a sensitivity of $100 \%$ in favouring malignancy.

The delayed phase is described as persistent, plateau or washout.17,21 Lesions with rapid or medium initial enhancement followed by a delayed plateau or washout have a positive predictive value of $77 \%$ for malignancy. In our study, continued or persistent pattern was essentially a feature of benign lesions. A sensitivity of $72 \%$ and specificity of $100 \%$, PPV of $100 \%$ and NPV of $81 \%$ was found with this pattern for benignity. Plateau pattern was indeterminate. Washout pattern showed sensitivity of $95.45 \%$, specificity of $94.44 \%$, PPV of $95.45 \%$ and NPV of $94.44 \%$ for malignancy. When cases with non-mass like enhancement were also included for calculations, sensitivity further decreased to $89.28 \%$.

The accuracy of the study in detecting malignant and benign lesions increases more when considering multiple parameters. Thus, specificity and PPV approaches to $100 \%$ to diagnose benign lesion when oval shape, smooth margin and type 1 curve is considered [Table 1]. Irregular shape spiculated margin and Type 3 curve shows a specificity and PPV of $100 \%$ for malignant lesions [Table 2]. In our study, the histopathologic report did not correlate well with the BIRADS categorisation. There was considerable overlap between IDCs and papillomas as well as benign intraductal hyperplasias. Lesions in B3 were all benign lesions. In various studies, the sensitivity of MRI to detect breast lesions were $94 \%-100 \%$ and specificity ranged from $37 \%$ - 97\%. In this study, the overall sensitivity was $78 \%$ in detecting malignancy and specificity $63 \%$.

\section{CONCLUSION}

MR imaging is more useful than mammography for invasive cancer, particularly in heterogeneously dense or extremely dense breasts. In non-fatty breasts, US and MR imaging were more sensitive than mammography for invasive cancer, but both MR imaging and US involved risk of overestimation of tumour extent. The enhancement of lymph nodes in the axilla and even (rarely) internal mammary chain was seen at MR imaging. It is found that when a node is identified at MR imaging that enhances more intensely or is visually strikingly larger than adjacent nodes or has lost its fatty hilum that subsequent identification and US-guided fine-needle aspiration biopsy can facilitate surgical planning.

In conclusion, the results of the present study confirm that the diagnostic yield of MR imaging in breast lesions is definitely superior to that of combined mammography and sonography. MR imaging is more sensitive to malignancy than any other single imaging modality. MR imaging is also useful in staging of malignancy, detecting multifocality and multicentricity of lesions and in detection of early recurrence in treated cases. However, in the present scenario, MR imaging cannot be routinely used as a screening test due to cost issues. Instead, MR imaging can be definitely used as a problem solving tool in situations where both mammography and sonomammography fail.

\section{REFERENCES}

[1] Mandelson MT, Oestreicher N, Porter PL, et al. Breast density as a predictor of mammographic detection: comparison of interval-and screen-detected cancers. J Natl Cancer Inst 2000;92(13):1081-7.

[2] Kolb TM, Lichy J, Newhouse JH. Comparison of the performance of screening mammography, physical examination, and breast US and evaluation of factors that influence them: an analysis of 27,825 patient evaluations. Radiology 2002;225(1):165-75.

[3] Berg WA. Rationale for a trial of screening breast ultrasound: American college of radiology imaging network (ACRIN) 6666. AJR Am J Roentgenol 2003;180(5):1225-8.

[4] Stomper PC, D'Souza DJ, DiNitto PA, et al. Analysis of parenchymal density on mammograms in 1353 women 25-79 years old. AJR Am J Roentgenol 1996;167(5):1261-5. 
[5] Wazer DE, Schmidt-Ullrich RK, Ruthazer R, et al. The influence of age and extensive intraductal component histology upon breast lumpectomy margin assessment as a predictor of residual tumor. Int J Radiat Oncol Biol Phys 1999;45(4):885-91.

[6] Liberman L, Morris EA, Dershaw DD, et al. MR imaging of the ipsilateral breast in women with percutaneously proven breast cancer. AJR Am J Roentgenol 2003;180(4):901-10.

[7] Fischer U, Kopka L, Grabbe E. Breast carcinoma: effect of preoperative contrast-enhanced MR imaging on the therapeutic approach. Radiology 1999;213(3):881-8.

[8] Orel SG, Schnall MD, Powell CM, et al. Staging of suspected breast cancer: effect of MR imaging and MRguided biopsy. Radiology 1995;196(1):115-22.

[9] Rieber A, Merkle E, Bohm W, et al. MRI of histologically confirmed mammary carcinoma: clinical relevance of diagnostic procedures for detection of multifocal or contralateral secondary carcinoma. J Comput Assist Tomogr 1997;21(5):773-9.

[10] Lee SG, Orel SG, Woo IJ, et al. MR imaging screening of the contralateral breast in patients with newly diagnosed breast cancer: preliminary results. Radiology 2003;226(3):773-8.

[11] Hlawatsch A, Teifke A, Schmidt M, et al. Preoperative assessment of breast cancer: sonography versus MR imaging. AJR Am J Roentgenol 2002;179(6):1493-501.

[12] Mai KT, Yazdi HM, Ford JC, et al. Predictive value of extent and grade of ductal carcinoma in situ in radiologically guided core biopsy for the status of margins in lumpectomy specimens. Eur J Surg Oncol 2000;26(7):646-51.

[13] Rodenko GN, Harms SE, Pruneda JM, et al. MR imaging in the management before surgery of lobular carcinoma of the breast: correlation with pathology. AJR Am J Roentgenol 1996;167(6):1415-9.

[14] Weinstein SP, Orel SG, Heller R, et al. MR imaging of the breast in patients with invasive lobular carcinoma. AJR Am J Roentgenol 2001;176(2):399-406.

[15] Teifke A, Lehr HA, Vomweg TW, et al. Outcome analysis and rational management of enhancing lesions incidentally detected on contrast enhanced MRI of the breast. AJR Am J Roentgenol 2003;181(3):655-62.

[16] Kuhl CK, Simon B, Sondermann E, et al. Ultra-high resolution breast MR imaging using intravascular contrast agent (gadomer17). Radiology 2002;225:652.

[17] Kuhl CK, Mielcareck P, Klaschik S, et al. Dynamic breast MR imaging: are signal intensity time course data useful for differential diagnosis of enhancing lesions. Radiology 1999;211(1):101-10.

[18] Liberman L, Morris EA, Benton CL, et al. Probably benign lesions at breast magnetic resonance imaging: preliminary experience in high-risk women. Cancer 2003;98(2):377-88.

[19] Krishnamurthy S, Sneige N, Bedi DG, et al. Role of ultrasound-guided fine-needle aspiration of indeterminate and suspicious axillary lymph nodes in the initial staging of breast carcinoma. Cancer 2002;95(5):982-8.

[20] Kerlikowske K, Grady D, Barclay J, et al. Effect of age, breast density, and family history on the sensitivity of first screening mammography. JAMA 1996;276(1):338.

[21] Kuhl CK, Klaschik S, Mielcarek P, et al. Do T2-weighted pulse sequences help with the differential diagnosis of enhancing lesions of dynamic breast MRI? J Magn Reson Imaging 1999;9(2):187-96. 